\title{
Research of Anti-Swing Control System of Tower Crane Based on EI Input Shaping
}

\author{
Xiang-Ao WANGa , ${ }^{*}$ Jin PENG and Xian-Bing WANG
}

School of Electronic and Electrical Engineering, Chuzhou University, Chuzhou 239000, P. R. China Corresponding author: Xiang-Ao WANG wxa@chzu.edu.cn*

Keywords: Index Terms-Tower crane, EI input shaping, Anti-swing.

\begin{abstract}
This thesis builds tower crane's simulation model using MATLAB/Simulink based on it's stress analysis, analyzes the design method of EI input shaper(Extra-Insensitive Input Shapers)in eliminating swing, and the simulation results of swing trajectory when doing heavy swing rotary motion were compared. Finally, the effectiveness of using EI input shaping controller is verified through experiment.
\end{abstract}

\section{Introduction}

As the rapid development of construction industry, the tower cranes are extensively used to lift the materials in construction sites. However, the problem is that the working efficiency are influenced by the longer lifting rope compared with the conventional industry crane. Although they swing the same angle, the swing range of the tower crane is larger than that of the conventional industry crane, which also cause the increase of the probability of collision during the motion. It is not effective to extinct the swaying [1] for tower crane by employing the conventional extinction-swing algorithm due to the differentness of mechanism system between the tower crane and the general bridge crane. The EI Input Shapers can be adaptive to more changeable situation, because of its non-sensitive to the system parameters and other advantages.

At present, researches about the bridge crane are popular [2-7], however the research of tower crane anti-swing is deficiency, especially during the rotation motion. This paper has constructed the simulation model of tower crane by using the Matlab software based on analysis of kinematics model, and analyzed the anti-swing benefit of the EI input shaper. Finally, the availability of this method is verified through experiment platform.

\section{Kinematics Model Analysis of the Tower Crane}

In order to simplify the analysis process, the tower crane is simplified as bellow [8-9]:

(1)The fixed pulley for lifting is simplified to suspension point.

(2) Regardless of the influence of the pulley block, air and wind resistance.

(3) Regardless of the weight of the steel wire rope.

As shown in Figure 1, the kinematics model of a weight ( $\mathrm{m}$ as the mass) in the inertial coordination $\operatorname{system}(\mathrm{X}, \mathrm{Y}, \mathrm{Z})$ is constructed.

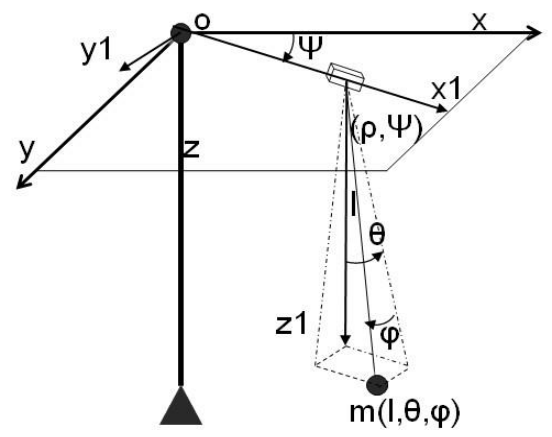

Figure 1. kinematics model of the tower crane 
The machine can rotate around $\mathrm{Z}$ axis. The coordinate $(\mathrm{x} 1, \mathrm{y} 1)$ is built. Provided the angle of $\mathrm{x} 1$ and $\mathrm{x}$ axis is $\Psi$, the orientation is decided by the right-hand rule, the length of jib is $\rho$, therefore the coordinate of the machine is $(\rho, \Psi)$. Provided the length of the rope is $1,(\rho, \Psi)$ as the origin of the spherical coordinate system is established, the coordinate of the weight is $(1, \theta, \varphi)$, therefore the vector quantity of the weight in the rotation coordination is

$$
\stackrel{1}{r}_{l}=(\rho+l \cos (\phi) \sin (\theta), l \sin (\phi), l \cos (\phi) \cos (\theta))
$$

The vector quantity of the machine is

$$
r_{t}=(\rho, 0,0)
$$

The speed of the machine and weight is derived through taking two vectors of time derivative. Provided the mass of the weight is $\mathrm{M}$, the rotated inertia of the whole system is $\mathrm{J}$, therefore the kinetic energy is

$$
T=\frac{1}{2} m\left(\frac{d r_{l}}{d t} \cdot \frac{d r_{l}}{d t}\right)+\frac{1}{2} M\left(\frac{d r_{t}}{d t} \cdot \frac{d r_{t}}{d t}\right)^{2}+\frac{1}{2} J\left(\frac{d}{d t} \psi\right)^{2}
$$
is

Provided the surface potential energy of xoy is zero, therefore the potential energy of the weight

$$
V=-m g l \cos (\phi) \cos (\theta)
$$

According to Lagrange function $\mathrm{L}=\mathrm{T}-\mathrm{V}$, the formula is created as

$$
\frac{d}{d t}\left[\frac{\partial L}{\partial\left(\frac{d}{d t} q_{i}\right)}\right]-\frac{\partial L}{\partial q_{i}}=Q_{i}
$$

In the formula (5), qi represents the four variables in the system of generalized coordinate $(\rho, \Psi, \theta, \varphi)$, Qi is the corresponding generalized force: the atress in the $\mathrm{x} 1$ direction is $\mathrm{F} \rho$, the torque in $\mathrm{z}$ direction is $\mathrm{T} \Psi$. Due to the gravity of the acting force of $\theta, \varphi$ are conservative forces, namely $\mathrm{Q} \theta=\mathrm{Q} \varphi=0$, the differential equation including two variables can be derived as

$$
\begin{aligned}
& l \frac{d^{2} \theta}{d t^{2}} \cos (\phi)+g \sin (\theta)-l \frac{d^{2} \Psi}{d t^{2}} \sin (\phi) \cos (\theta)- \\
& \rho\left(\frac{d \psi}{d t}\right)^{2} \cos (\theta)+l\left(\frac{d \psi}{d t}\right)^{2} \cos (\phi) \sin (\theta) \cos (\theta)- \\
& 2 l \frac{d \theta}{d t} \frac{d \phi}{d t} \sin (\phi)-2 l \frac{d \psi}{d t} \frac{d \phi}{d t} \cos (\phi) \cos (\theta)=0 \\
& l \frac{d^{2} \phi}{d t^{2}}+g \sin (\phi) \cos (\theta)-\frac{d^{2} \rho}{d t^{2}} \sin (\phi) \sin (\theta)+ \\
& l \frac{d^{2} \psi}{d t^{2}} \sin (\theta)+\frac{d^{2} \psi}{d t^{2}} \rho \cos (\phi)+l\left(\frac{d \theta}{d t}\right)^{2} \cos (\phi) \sin (\phi) \\
& +\left(\frac{d \psi}{d t}\right)^{2} \rho \sin (\phi) \sin (\theta)-l\left(\frac{d \psi}{d t}\right)^{2} \sin (\phi) \cos (\theta)^{2} \cos (\phi)+ \\
& 2 \frac{d \rho}{d t} \frac{d \psi}{d t} \cos (\phi)+2 l \frac{d \theta}{d t} \frac{d \psi}{d t} \cos (\phi)^{2} \cos (\theta)=0
\end{aligned}
$$

The above formulas which are interconnection and non-liner need to be linearized to obtain the swing equation by neglecting the lesser part of the linearized formula as

$$
l \frac{d^{2} \theta}{d t^{2}}+\left[g+l\left(\frac{d \psi}{d t}\right)^{2}\right] \theta-2 l \frac{d \psi}{d t} \frac{d \varphi}{d t}-l \frac{d^{2} \psi}{d t^{2}} \varphi=-\frac{d^{2} \rho}{d t^{2}}+\rho\left(\frac{d \psi}{d t}\right)^{2}
$$




$$
l \frac{d^{2} \varphi}{d t^{2}}+\left[g-l\left(\frac{d \psi}{d t}\right)^{2}\right] \varphi+2 l \frac{d \theta}{d t} \frac{d \psi}{d t}+l \theta \frac{d^{2} \psi}{d t^{2}}=-\rho \frac{d^{2} \psi}{d t^{2}}-2 \frac{d \rho}{d t} \frac{d \psi}{d t}
$$

The above formula is the swing equation of the weight on the tower crane[10], which meets the requirements in the little swing range. In the formula, the parameters $\theta$ and $\varphi$ are only related to the length of the rope.

\section{Control Method of EI Input Shaper}

The time-domain expression of $\mathrm{n}$ pulses input shaper is

$$
G(t)=\sum_{i=1}^{n} A_{i} \delta\left(t-t_{i}\right)
$$

After the role of the input shaper $(t>t n)$, the ratio of the unitary pulse response in the system to that without input shaper controlling is called residual vibration, the formula is

$$
V(\omega, \xi)=e^{-\xi \omega t_{n}} \sqrt{C(\omega, \xi)^{2}+S(\omega, \xi)^{2}}
$$

In the formula

$$
\begin{gathered}
C(\omega, \xi)=\sum_{i=0}^{n-1} A_{i} e^{\xi \omega t_{i}} \sin \left(\omega \sqrt{1-\xi^{2}} t_{i}\right) \\
S(\omega, \xi)=\sum_{i=0}^{n-1} A_{i} e^{\xi \omega t_{i}} \cos \left(\omega \sqrt{1-\xi^{2}} t_{i}\right)
\end{gathered}
$$

Provided Vexp is the permissible value of the residual vibration range with undampted natural frequency of the system, and the residual vibration ranges are both zero when the frequency $\omega \mathrm{h}$ and $\omega l$ are a bit higher or lower than the natural one, therefore, the above formula can meet the requirement in $[\omega \mathrm{h}, \omega \mathrm{l}]$. The three designed EI pulse sharper models as [11]

$$
\left\{\begin{array} { l } 
{ t _ { 1 } = 0 } \\
{ t _ { 2 } = \frac { T } { 2 } } \\
{ t _ { 3 } = T }
\end{array} \left\{\begin{array}{l}
A_{1}=\frac{1+V_{\text {exp }}}{4} \\
A_{2}=\frac{1-V_{\text {exp }}}{2} \\
A_{3}=\frac{1+V_{\text {exp }}}{4}
\end{array}\right.\right.
$$

Therefore

$$
\left\{\begin{array}{l}
\omega_{h}=\omega+\Delta \omega \\
\omega_{l}=\omega-\Delta \omega \\
\frac{\Delta \omega}{\omega}=\frac{\arccos \left(\frac{1-V_{\text {exp }}}{1+V_{\text {exp }}}\right)}{\pi} \\
T=\frac{2 \pi}{\omega \sqrt{1-\xi^{2}}}
\end{array}\right.
$$

Provided $\xi=0$, the period of vibration is calculated by the formula as

$$
T=\frac{2 \pi}{\omega}=2 \pi \sqrt{\frac{l}{g}}
$$

Where $l$ is the length of the rope. 


\section{Simulation and the Test Result}

Taking advantage of the powerful simulation function of the MATLAB/Simulink, the simulation model shown in Figure 2 is constructed according to the vibration formula analyzed, at the same time, the outputting signal waveforms are edited by using signalbilder module. Provided $\rho=50 \mathrm{~m}$ is the jib length with static condition, the length of the rope 1 is equal to $50 \mathrm{~m}$, therefore the highest angular velocity of rotation $\omega$ is $0.03 \mathrm{rad} / \mathrm{s}$, the acceleration and deceleration of time are both 6 seconds.

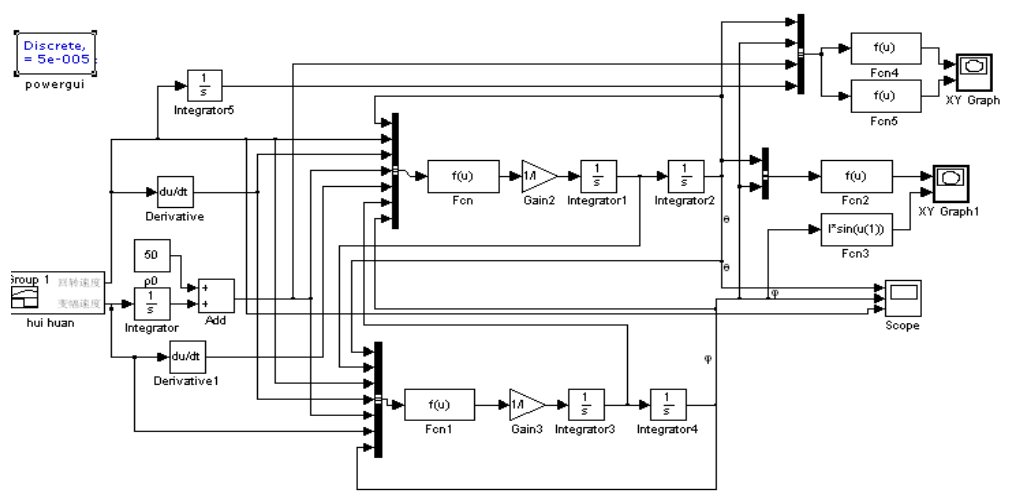

Figure 2. Simulation model of tower crane's control system

In the case that the tower crane starts to rotate uniformly for $114 \mathrm{~s}$ from $5 \mathrm{~s}$ after accelerating to the highest rotation velocity, and then decelerates to rotate until stopping, the total time of motion is $126 \mathrm{~s}$. The simulation is carried out with EI shaper and without EI shaper respectively. The alteration of $\theta$ and $\varphi$ waveforms are shown in Figure 3 and Figure 4.

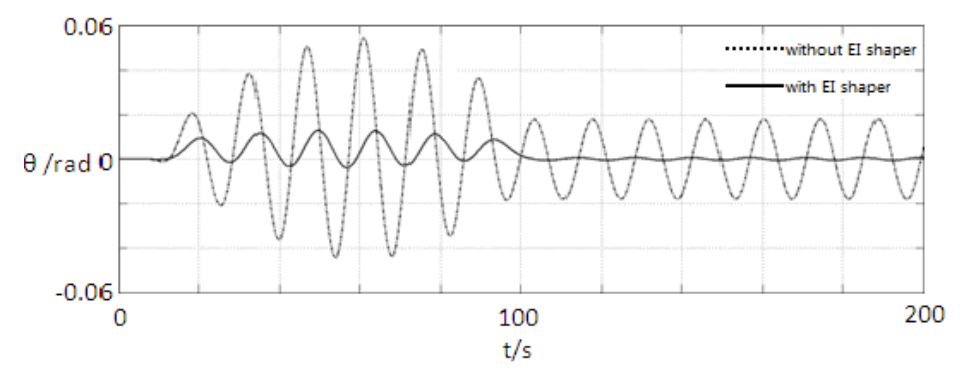

Figure 3. The comparison of $\theta$ waveforms gained with EI shaper and without EI shaper

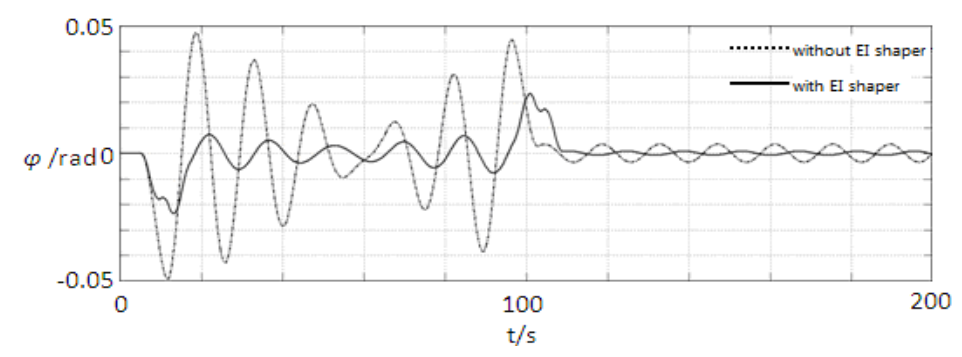

Figure 4. The comparison of $\varphi$ waveforms gained with EI shaper and without EI shaper

From the simulation waveforms without EI shaper shown in the figures, both $\theta$ and $\varphi$ change periodically and superpose a low frequency vibration during the rotation motion, the frequency is swing frequency and rotation frequency respectively. As the rotation angle changing, $\theta$ and $\varphi$ influence each other. The maximum value of $\theta$ is gained when the jib of tower crane rotates $\pm 90^{\circ}$, and that maximum value of $\varphi$ can be obtained when the jib of tower crane rotates $0^{\circ}$ or $180^{\circ}$. The difference is caused by the superposition of rotation motions due to the mutually perpendicular swing. The amplitude of $\theta$ and $\varphi$ decreases largely after adding EI shaper, and meet the requirement that the 
residual swing is less than $10 \%$. These demonstrate that the method is available.

In order to vertify the effectiveness of EI shaper, a tower crane model is constructed as the experiment platform, the jib length is 2 meters and the highest rotation velocity is $2 \mathrm{r} / \mathrm{min}$ as shown in Figure 5.

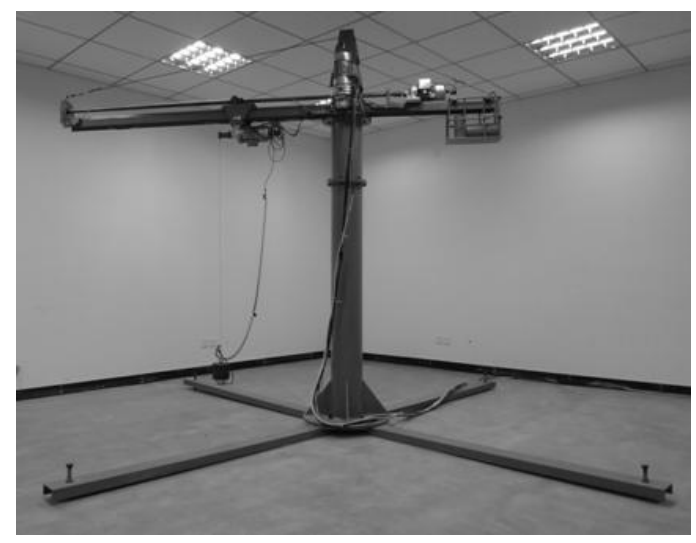

Figure 5. The experiment platform of tower crane

The frequency transducer BEM520 series specail for lifting purpose is used in the experiment platform, the preset frequency is $100 \mathrm{~Hz}$, which is corresponding to the highest rotated velocity, the rotated time is 6 seconds, both acceleration and deceleration time are 0.8 seconds. The micromechanical voltage-type gyroscope MEMS is adopted to inspect the angles, which can measure the angular velocity in two directions at the same time, and then calculate the angle by integration. The upper computer constructed by Labview 2012 completes the data acquisition and draws the swing waveform. Figure 6 and Figure 7 show respectively the swing waveforms of $\theta$ and $\varphi$ after and before using EI shaper.

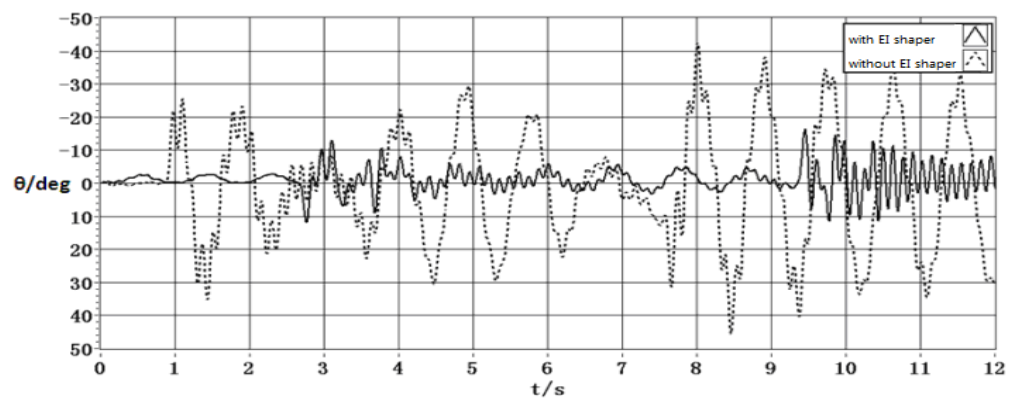

Figure 6. Alteration of the $\theta$ waveforms with EI shaper and without EI shaper

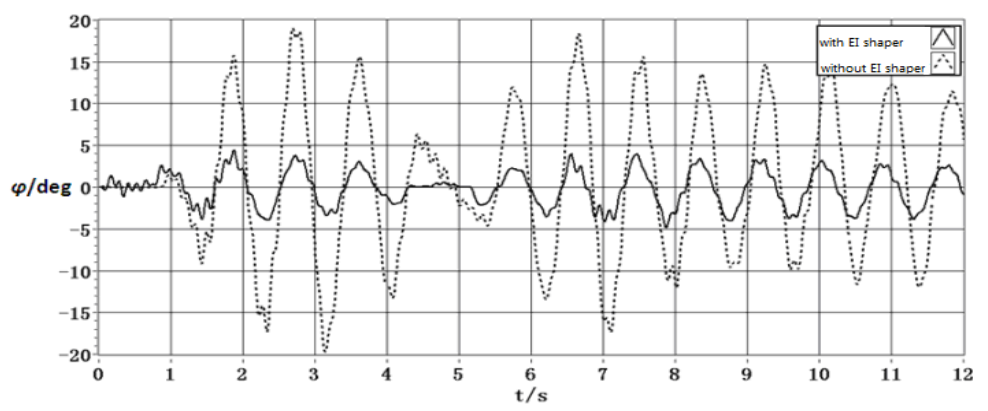

Figure 7. Alteration of the $\varphi$ waveforms with EI shaper and without EI shaper

As the Figure 6 and Figure 7 shown, it is clear that the angle of rotation can effect the amplitude of $\theta$ and $\varphi$ without using the EI shaper, which is similar as the simulation result, and the correctness of the simulation is proved by the alternating appearance of amplitude of $\theta$ and $\varphi$. However, the swing 
angle decreases significantly while adding the EI shaper. The tested waveform possesses much tiny vibration due to the mechanical vibration of the experiment platform.

\section{Conclusions}

In this paper, the equitation of rotation motion of the tower crane is derived based on the Lagrangian function. The simulation model of the tower crane is created by the MATLAB/Simulink, and be carried out through the given signal, which is set according to the speed of the rotation mechanism edited by the EI input shaping method. The swing situation of the weight is discussed with and without EI input shaper condition. Finally, the availability of anti-swing of the EI input shaper in rotation motion is vertified by the experiment platform of tower crane in laboratory, these provide a reference for practical engineering application.

\section{Acknowledgment}

This work was supported by the Natural Science Research of Education Department of Anhui Province under Grant KJ2015B11, the Key projects of excellent talent fund of Anhui Province under Grant 2013SQRL093ZD, the Project of Natural Science Foundation of Anhui Province under Grant 1308085QE97 and the Enterprise funding projects under Grant HX2016012.

\section{References}

1. L. Pao, W. E. Singhose, IFAC World Cong, (1996).

2. T. S. Wu, M. Karkoub, W. S Yu, C. T Chen, M. G. Her, Fuzzy Sets \& Sys, 290, 29 (2015)

3. M. S. Park, D. Chwa, S. K. Hong, IEEE Traons Ind Elec 55, 13 (2008)

4. L. J, W. E. Singhose, Vib and Aco 132, 10 (2010)

5. M. F. Daqaq, Z. N. Masoud, Nonlinear Dynam 45, 22 (2006)

6. H. Park, Chwa, K. Hong, Ctrl, Auto \& Sys 5, 9 (2007)

7. W. E. Singhose, P. L, Kenison M, Ctrl Eng Pra 21, 8 (2000)

8. Q. H. Ngo, K. S. Hong, Iet Ctrl Theor \& App 6, 7 (2012)

9. J. Vaughan, A. Yano, W. E. Singhose, Sound \& Vib 315, 19 (2008)

10. D. Blackburn, W. E. Singhose, J. Kitchen, V. Patrangenaru, J. Lawrence, The 8th Int'l Conf on Motion \& Vib Ctrl, 11 (2006)

11. Z. N. Masoud, M. F. Daqaq, IEEE Trans on Ctrl Sys Tech 14, 8 (2006) 